\title{
Immunoassay and $\mathrm{Nb2}$ lymphoma bioassay prolactin levels and mammographic density in premenopausal and postmenopausal women the Nurses' Health Studies
}

\author{
Megan S Rice ${ }^{1,2}$, Shelley S Tworoger ${ }^{1,2}$, Kimberly A Bertrand ${ }^{1,2}$, Susan E Hankinson ${ }^{1,2,3}$, \\ Bernard A Rosner ${ }^{1}$, Yvonne B Feeney ${ }^{4}$, Charles V Clevenger ${ }^{5}$, and Rulla M Tamimi ${ }^{1,2}$ \\ ${ }^{1}$ Channing Division of Network Medicine, Brigham and Women's Hospital and Harvard Medical \\ School, Boston, MA, USA \\ ${ }^{2}$ Department of Epidemiology, Harvard School of Public Health, Boston, MA, USA \\ ${ }^{3}$ Division of Biostatistics and Epidemiology, University of Massachusetts, Amherst, MA, USA \\ ${ }^{4}$ Department of Pathology, Northwestern University, Chicago, Illinois USA \\ ${ }^{5}$ Department of Pathology, Virginia Commonwealth University, Richmond, VA
}

\begin{abstract}
Background-Higher circulating prolactin levels have been associated with higher percent mammographic density among postmenopausal women in some, but not all studies. However, few studies have examined associations with dense area and non-dense breast area breast or considered associations with prolactin $\mathrm{Nb} 2$ lymphoma cell bioassay levels.
\end{abstract}

Methods-We conducted a cross-sectional study among 1124 premenopausal and 890 postmenopausal women who were controls in breast cancer case-control studies nested in the Nurses' Health Study (NHS) and NHSII. Participants provided blood samples in 1989-1990 (NHS) or 1996-1999 (NHSII) and mammograms were obtained from around the time of blood draw. Multivariable linear models were used to assess the associations between prolactin levels (measured by immunoassay or bioassay) with percent density, dense area, and non-dense area.

Results-Among 1124 premenopausal women, percent density, dense area, and non-dense area were not associated with prolactin immunoassay levels in multivariable models ( $\mathrm{p}$-trends $=0.10$, 0.18 , and 0.69 , respectively). Among 890 postmenopausal women, those with prolactin immunoassay levels in the highest versus lowest quartile had modestly, though significantly, higher percent density (difference $=3.01$ percentage points, $95 \% \mathrm{CI}$ : $0.22,5.80$ ) as well as lower non-dense area (p-trend=0.02). Among women with both immunoassay and bioassay levels, there

Corresponding author: Megan S Rice, ScD, 181 Longwood Ave $3^{\text {rd }}$ Floor, Boston, MA 02115, Tel: (617) 525-2039, Fax: (617) 525-2008,nhmsr@channing.harvard.edu.

Conflict of interest: None of the authors has a competing interest to declare.

Author contributions

MSR participated in the design of the study, conducted the statistical analysis, and drafted the manuscript. SST, BR, SHE, and RMT participated in the design of the study, advised on the statistical analysis, and drafted the manuscript. KAB designed and conducted the mammographic density recalibration. YBF and CVC conducted the measurements of prolactin. All authors read, contributed to, and approved the final manuscript. 
were no consistent differences in the associations with percent density between bioassay and immunoassay levels.

Conclusions-Postmenopausal women with prolactin immunoassay levels in the highest quartile had significantly higher percent density as well as lower non-dense area compared to those in the lowest quartile.

Impact-Future studies should examine the underlying biologic mechanisms, particularly for non-dense area.

\section{Keywords}

Mammographic density; breast cancer risk; prolactin; epidemiology; plasma biomarkers

\section{Introduction}

Mammographic density is the radiographic appearance of the breast on a mammogram and is a strong predictor of subsequent breast cancer risk [1]. Epithelial and stromal tissue in the female breast is radiodense and appears light on a mammogram, whereas fat is radiolucent and appears dark. Women with over 75 percent dense tissue on a mammogram have 4-6 times the risk of breast cancer compared to women with little to no dense tissue [1,2]. Mammographic density is associated with a number of reproductive factors, including parity, and is thought to represent cumulative exposure to hormones.

Prolactin, a hormone involved in the normal differentiation of mammary epithelium, may influence breast cancer risk through cellular proliferation and survival [3]. Higher circulating prolactin levels have been associated with higher mammographic density among postmenopausal women in some [4, 5], but not all studies. [6, 7] For example, in a previous analysis of 489 postmenopausal women in the Nurses' Health Study (NHS), there was no significant association between prolactin and percent mammographic density. [8] The few studies in premenopausal women have not observed an association. $[4,7,9]$ In addition, only two studies examined the association with non-dense area of the breast $[9,10]$, with one study reporting an inverse association with prolactin levels among postmenopausal women. [10] This area is of particular interest as the dense area and non-dense area of the breast may have independent effects on breast cancer risk. [11, 12]

Prior studies have determined prolactin levels using an immunoassay that measures multiple forms of circulating prolactin. However, this immunoassay may not reflect levels of bioactive prolactin, as prolactin isoforms have varying biological activities. [13, 14] The $\mathrm{Nb} 2$ lymphoma cell bioassay is a sensitive measure of somatolactogenic activity of prolactin in plasma [15]; however data on prolactin bioassay levels and breast cancer or breast density are limited [16]. Therefore, the purpose of this cross-sectional study was to examine the association between circulating prolactin levels, as measured by the Nb2 lymphoma cell bioassay, with measures of mammographic density among both premenopausal and postmenopausal women in the NHS and NHSII. Further, we updated our previous analysis of prolactin immunoassay levels and percent density to include over 1100 premenopausal women as well as approximately 400 additional postmenopausal women. Further, this 
updated analysis also examines the relationship between prolactin levels with dense area and non-dense area of the breast.

\section{Materials and Methods}

\section{Study population}

The NHS cohort began in 1976 when 121,700 female registered nurses, 30 to 55 years of age and residing in 11 U.S. states completed an initial questionnaire. In 1989, 116,430 female registered nurses, aged 25 to 42, from 14 U.S. states completed an initial questionnaire forming the NHSII cohort. Both cohorts are followed by biennially mailed questionnaires to collect information on exposures and covariates as well as incident diseases.

In 1989-1990, 32,826 NHS participants, ages 43 to 70, provided blood samples. Between 1996 and 1999, we obtained blood samples from 29,611 NHSII members, aged 32-45 years. Characteristics of both cohorts and blood collection details have been described previously [17-20]. Briefly, in the NHSII, premenopausal women who had not taken any type of hormones, been pregnant, or breastfed in the previous six months $(n=18,521)$, provided timed blood samples on the third to fifth day of their menstrual cycle (follicular sample) and seven to nine days before the anticipated start of their next cycle (luteal sample). Follicular plasma was aliquoted by the participant between eight and 24 hours after collection and frozen. All NHS participants as well as NHSII women who were ineligible to provide timed samples (i.e., perimenopausal, postmenopausal, had a simple hysterectomy, currently used oral contraceptives or other hormones, or declined to give timed samples; $n=11,090$ ) provided a single blood sample (referred to as "untimed" samples). For both timed and untimed samples, women shipped the blood to our laboratory, with an ice-pack, via overnight courier, where the samples were processed, separated into plasma, red blood cell, and white blood cell components, and aliquoted into labeled cryotubes.

We restricted our analysis to women who were controls in the NHS or NHSII nested breast cancer case-control studies and were matched to cases diagnosed between 1990 and 2004 (NHS) or 1999 and 2007 (NHSII) [17, 21]. Breast cancer cases were matched to one or two controls on age, menopausal status at blood draw and diagnosis, current postmenopausal hormone use (PMH), month, time of day, fasting status at time of blood collection, and luteal day (NHSII timed samples only). We collected mammograms conducted as close as possible to the date of blood collection for women in the nested breast cancer case-control studies. Among controls in the NHS and NHSII case-control studies, 2939 had measures of prolactin immunoassay levels, mammographic density, and body mass index (BMI). We next excluded 5 controls whose prolactin immunoassay levels were determined to be outliers based on the generalized extreme studentized deviate many-outlier detection approach [22]. We further restricted our study population to the 1124 women who were premenopausal at both blood collection and mammogram as well as 890 women who were postmenopausal at both blood collection and mammogram and who reported no current PMH use. The median time between mammography and blood collection was 9 months for premenopausal women and 13 months for postmenopausal women. As part of another nested study of prolactin Nb2 bioassay levels and breast cancer risk, 551 of these women (317 premenopausal and 234 
postmenopausal) had prolactin bioassay levels measured. The study was approved by the Committee on the Use of Human Subjects in Research at the Brigham and Women's Hospital.

\section{Laboratory assays}

Prolactin was assayed by microparticle enzyme immunoassay in 12 batches for untimed samples in the NHS and in 3 batches for luteal, follicular and untimed samples in the NHSII. We averaged the prolactin levels in follicular and luteal samples among women in the NHSII who provided timed samples. Samples were assayed at the Reproductive Endocrinology Unit Laboratory at the Massachusetts General Hospital using the AxSYM Immunoassay system (Abbott Diagnostics, Chicago, IL) as well as by Christopher Longcope, MD (University of Massachusetts Medical Center, Worcester, MA), using the IMx System (Abbott Laboratory, Abbott Park, IL). The correlation between the two laboratories was 0.91 and across different batches within the same data set was greater than 0.95 . The limit of detection was $0.6 \mathrm{ng} / \mathrm{mL}$. Nb2 was assayed in the NHS in 65 batches and in the NHSII in 39 batches, for a total of 104 batches consisting of approximately 25-30 women each. The $\mathrm{Nb} 2$ bioassay for biologically active somatolactogen was performed in duplicate as previously described. [15, 23] To assess prolactin variability, $10 \%$ blinded replicate quality control (QC) samples were randomly interspersed across the entire set of participant samples. All immunoassay batches had QC samples; however due to the smaller batch size of the bioassay, 77 of the 104 bioassay batches included blinded QC samples. Average within batch coefficients of variation $(\mathrm{CVs})$ were less than $12 \%$ for the immunoassay. For the bioassay, within batch CVs were calculated either using blinded QC samples ( $\mathrm{n}=77$ batches) or using the average $\mathrm{CV}$ of the duplicate participant samples within a batch if blinded QCs were not available ( $\mathrm{n}=27$ batches). For 97 of the batches, CVs ranged from $0.3-17.3 \%$ (mean=7.0\%). Seven batches had CVs above 20\% (range, 21.5-26.6). Therefore, we conducted a sensitivity analysis excluding these 7 batches. The between batch CV for the bioassay was $40 \%$.

\section{Mammographic density}

The craniocaudal views of both breasts were digitized with a Lumysis 85 laser film scanner. Using the Cumulus software for computer-assisted thresholding, we measured absolute dense area, absolute non-dense area (the total area minus the dense area), and percent dense area (the dense area divided by the total area) and averaged the density parameters of both breasts. Images from NHS participants were read in two batches by two readers. In NHSII, a single observer read the mammograms in two batches, three years apart with a small number of mammograms included in both batches. While there was high reproducibility within each batch, there was evidence of between batch variability in the NHSII. Therefore, for the overall NHSII breast cancer case-control mammography dataset, we used multivariable linear regression models to estimate the effect of batch on density measurements, controlling for age, menopausal status, BMI, and case-control status [18]. We then adjusted density measurements in the second NHSII batch by adding the coefficient for mammogram batch to the raw value to estimate the measurements that would have been obtained if the mammogram had been included in the first batch. For all batches, readers were blinded to case-control status. 


\section{Covariate data}

We used the covariate information from the biennial questionnaire preceding the mammogram date to obtain data on the following covariates: BMI $\left(\mathrm{kg} / \mathrm{m}^{2}\right)$, history of benign breast disease (BBD), family history of breast cancer, age at menarche, age at first birth and parity, breastfeeding, smoking, alcohol intake, past PMH use, and age at menopause.

\section{Statistical analysis}

Due to batch-to-batch variation over time, we recalibrated prolactin immunoassay and bioassay levels from all batches to have a comparable distribution to an average batch according to methods outlined by Rosner et al [24]. Using this method, we assumed that all batches combined represented an average batch. We then regressed prolactin immunoassay or bioassay levels on indicator variables for batch as well as the following covariates: age, BMI, fasting status, time of blood collection, parity, history of PMH use, and use of antidepressants at the time of blood collection. Prolactin immunoassay and bioassay levels were then recalibrated based on the coefficients for each batch and the average of the batch coefficients. We classified the recalibrated values into quartiles based on the overall distribution.

We used generalized linear models adjusting for matching factors to evaluate the association of prolactin immunoassay and bioassay levels with mammographic density. These models accounted for any correlation between controls who were matched to the same case. We modeled square-root transformed dense area and non-dense area on mammogram as outcomes since both distributions were skewed. We estimated the difference from the lowest quartile of percent density, square-root dense area, and square-root non-dense area for each quartile of prolactin levels. We tested for trend using Wald tests by ordinally modeling the median of the quartiles. Our primary model adjusted for age (continuous) and matching factors with subsequent models additionally adjusting for correlates of prolactin and breast density including history of BBD, family history of breast cancer, age at menarche, age at first birth, parity, breastfeeding, antidepressant use, smoking status, alcohol use, BMI, as well as past PMH use and age at menopause (among postmenopausal women). To determine if the associations between prolactin levels and percent density varied by age $(<45,45+$ years for premenopausal; $<60,60+$ for postmenopausal), BMI $\left(<25,25+\mathrm{kg} / \mathrm{m}^{2}\right)$, cohort (NHS, NHS2; in premenopausal women) and sample type (timed, untimed; in premenopausal women), we calculated Wald tests for the interaction term between the ordinal median variable for the hormone and the binary age, BMI, cohort, and sample type variables. In addition, we conducted a secondary analysis restricting our analysis of prolactin immunoassay levels to the 551 women who also had $\mathrm{Nb} 2$ bioassay levels measured to allow a better comparison between the two assays. Lastly, in sensitivity analyses, we restricted to parous women, restricted to NHS women among postmenopausal women, and excluded 7 batches with $\mathrm{CVs}>20 \%$ in our analysis of prolactin $\mathrm{Nb} 2$ bioassay levels. We considered a two-sided p-value of less than 0.05 to be statistically significant and used SAS 9.2 software (SAS Institute, Cary, NC, USA) for all analyses. 


\section{Results}

For both pre- and postmenopausal women, those in the highest category of percent mammographic density were younger, had a lower BMI, were more likely to be nulliparous, and were more likely to have a history of BBD compared to women in the lowest quartile of mammographic density. (Table 1)

In multivariable models, we did not observe a statistically significant association between prolactin immunoassay levels and percent mammographic density, dense area, or non-dense area in the 1124 premenopausal women (p-trends $\bigotimes_{0.10}$ ) (Table 2). For example, in our fully adjusted model, women in the highest quartile of prolactin levels had 2.24 percentage points higher percent mammographic density than women in the lowest quartile (95\%CI:-0.79, 5.27, p-trend=0.10). Among 890 postmenopausal women, those in the highest quartile of prolactin levels had significantly higher percent mammographic density compared to those in the lowest quartile of levels (difference $=3.0195 \%$ CI: $0.22-5.08$ ), however the trend across quartiles did not reach statistical significance (p-trend-0.07). Interestingly, prolactin immunoassay levels were significantly inversely associated with non-dense area (ptrend=0.02), but not with dense area ( $\mathrm{p}$-trend=0.40).

Next, we examined the association between prolactin and mammographic density among the 551 women with prolactin measured by both the immunoassay and $\mathrm{Nb} 2$ bioassay. Among 317 premenopausal women, both prolactin immunoassay and bioassay levels were positively associated with percent density, though the difference between extreme quartiles of prolactin was significant only for immunoassay levels. Women in the highest quartiles of prolactin levels had 6.51 (95\%CI: 0.19, 12.82, p-trend=0.05) and $3.85(-2.23,9.93$, p-trend=0.22) percentage point higher density compared to women in the lowest quartiles, for the immunoassay and bioassay respectively (Table 3). The association between bioassay levels and percent density in premenopausal women was somewhat stronger when we restricted to bioassay batches with CVs $<20 \%(\mathrm{~N}=296$; comparable difference $=5.79$ percentage points, 95\%CI:-0.56, 12.15; p-trend=0.08). There was no significant association between prolactin immunoassay or $\mathrm{Nb} 2$ bioassay levels and dense or non-dense area (p-trends $\searrow 0.09$, respectively). Among 234 postmenopausal women, there was no association between prolactin immunoassay levels and percent density, dense area, or non-dense area (ptrend $=0.89,0.70$, and 0.79 , respectively) (Table 4 ). In contrast, there was a significant inverse association with non-dense area $(\mathrm{p}$-trend $=0.01)$ as well as a non-significant positive association between prolactin $\mathrm{Nb} 2$ bioassay levels and percent density (p-trend $=0.06$ ) among these same women. Women in the highest quartile of prolactin $\mathrm{Nb} 2$ bioassay levels had 4.80 percentage points higher mammographic density compared to women in the lowest quartile (95\% CI: -1.81,11.42). However, the association between bioassay levels and percent density in postmenopausal was stronger when we restricted to the batches with CVs $<20 \%$ $(\mathrm{N}=220$; comparable difference $=5.60,95 \% \mathrm{CI}:-0.96,12.16$; $\mathrm{p}$-trend $=0.03)$. The association between prolactin immunoassay levels and percent density did not vary by age for either premenopausal $(\mathrm{p}$-interaction $=0.22$ ) or postmenopausal women $(\mathrm{p}$-interaction $=0.61$ ) nor by BMI for either premenopausal (p-interaction $=0.29$ ) or postmenopausal women (pinteraction $=0.45$ ). Further, the association did not differ by cohort (p-interaction $=0.23$ ) or sample type (p-interaction=0.35) among premenopausal women. Lastly, the association 
among postmenopausal women was similar when we excluded NHS2 postmenopausal women (e.g., difference between extreme quartiles $=3.02,95 \%$ CI: $0.21,5.84$, p-trend $=0.06$ ). Overall, the observed associations were similar when we restricted to parous women (data not shown).

\section{Discussion}

In our analysis of over 1100 premenopausal women, there was no association between prolactin immunoassay levels and mammographic density. Among 890 postmenopausal women, we observed higher percent density among women in the highest quartile of prolactin immunoassay levels as well as an inverse association between immunoassay levels and non-dense area. In a subset of 234 postmenopausal women, there was a non-significant positive association between percent density and prolactin levels measured by the $\mathrm{Nb} 2$ lymphoma cell bioassay, but not for prolactin levels measured by the immunoassay. Our results are consistent with several studies that have observed no association between prolactin immunoassay levels and percent density, dense area, or non-dense area among premenopausal women $[4,7,9]$. In our previous analysis of 489 postmenopausal women in the NHS, we observed that women in the highest quartile of prolactin levels had a nonsignificant 3.7 percentage point higher percent density [25]. In our updated analysis, the association between prolactin immunoassay levels and percent density was similar (difference of 3.0), but statistically significant due to the increased sample size. These results are consistent with two studies that observed a positive association between prolactin immunoassay levels and percent mammographic density among postmenopausal women [4, 5]. However two other large studies $(\mathrm{N}=712$ and 802) reported no association [6, 7]. Differences between studies may be in part be related to the use of different assays, different sample types (e.g., serum vs plasma), and inclusion of women on PMH at blood collection and/or mammogram in some prior studies.

To our knowledge, only one study has examined the association between prolactin levels and non-dense area among postmenopausal women. Consistent with our study, McCormack et al. observed that higher prolactin levels were significantly associated with lower nondense area, but not with dense area [10]. These findings are contrary to our hypothesis that higher prolactin levels would be associated with an increase in dense area due to the proliferative effects of prolactin on epithelial cells. Further research is necessary to confirm the potential inverse association with non-dense area as well as to understand the mechanism by which prolactin may affect the amount of fat in the breast.

There was no consistent difference in the association with percent density between prolactin immunoassay and bioassay levels. In premenopausal women, the difference between extreme quartiles was similar between the immunoassay and the bioassay after we restricted to bioassay batches with $\mathrm{CV}$ s below $20 \%$. In postmenopausal women, the association with percent density was somewhat stronger for the bioassay compared to the immunoassay, especially after excluding bioassay levels from batches with high CVs. In two small breast cancer case-control studies the difference in prolactin levels between cases and controls was greater for the bioassay than for the immunoassay [26, 27]. While these prior studies suggest that the $\mathrm{Nb} 2$ lymphoma cell bioassay may measure the more biologically relevant portion of 
circulating prolactin, we did not observe consistent differences between the immunoassay and the bioassay with mammographic density or with breast cancer risk in the full nested case-control study [16], highlighting the need for additional research.

Our study has several limitations. While the CVs for the prolactin immunoassay are low there is likely some measurement error. This measurement error should be non-differential as the laboratory is blinded to density measurements. Though the CVs for some bioassay batches were high, we conducted a sensitivity analysis excluding batches with higher or unknown CVs. We only have a single measurement of prolactin levels, which may not be representative of long-term levels. However the three-year intra-class correlation coefficient (ICC) for the average of prolactin levels for follicular and luteal samples for both the immunoassay and bioassay was 0.64 among premenopausal NHSII women. [16, 28] Among 79 postmenopausal women in the NHS, the three-year ICC was 0.53 for prolactin immunoassay levels [29] and among 68 postmenopausal women the NHS the ICC was 0.63 for the bioassay over three years. [16] These data suggest that a single measurement of prolactin is representative of levels over at least a three-year period. Mammographic density measurements are highly reproducible though there is the potential for some random error. Since the mammogram reader was blinded to prolactin levels any measurement error should be non-differential. The strengths of our study include the centralized collection and reading of mammograms, high quality prolactin assays, and detailed adjustment for correlates of mammographic density.

\section{Conclusion}

Overall, this study suggests that prolactin levels are associated with modestly higher percent mammographic density and lower non-dense area among postmenopausal women.

Additional research into the association between prolactin and non-dense area of the breast may help elucidate the role of prolactin in breast carcinogenesis.

\section{Acknowledgments}

Funding/Support: This study was supported by research grants R01 CA138580, CA49449, CA67262, CA50385, CA131332, CA124865, P01 CA87969, T32 CA09001, R25 CA098566, and the Breast Cancer Research Foundation. We would like to thank the participants of the Nurses' Health Study and Nurses' Health Study II for their continuing contributions. We thank the following state cancer registries for their help: AL, AZ, AR, CA, CO, CT, DE, FL, GA, ID, IL, IN, IA, KY, LA, ME, MD, MA, MI, NE, NH, NJ, NY, NC, ND, OH, OK, OR, PA, RI, SC, TN, TX, VA, WA, WY.

\section{Abbreviations}

$\begin{array}{ll}\text { NHS } & \text { Nurses' Health Study } \\ \text { BMI } & \text { body mass index } \\ \text { BBD } & \text { benign breast disease } \\ \text { CV } & \text { coefficient of variation } \\ \text { ICC } & \text { intraclass correlation coefficient }\end{array}$




\section{References}

1. Byrne C, et al. Mammographic features and breast cancer risk: effects with time, age, and menopause status. J Natl Cancer Inst. 1995; 87(21):1622-1629. [PubMed: 7563205]

2. Boyd NF, et al. Quantitative classification of mammographic densities and breast cancer risk: results from the Canadian National Breast Screening Study. J Natl Cancer Inst. 1995; 87(9):670-675. [PubMed: 7752271]

3. Tworoger SS, Hankinson SE. Prolactin and breast cancer etiology: an epidemiologic perspective. J Mammary Gland Biol Neoplasia. 2008; 13(1):41-53. [PubMed: 18246319]

4. Boyd NF, et al. The association of breast mitogens with mammographic densities. Br J Cancer. 2002; 87(8):876-882. [PubMed: 12373602]

5. Greendale GA, et al. Serum prolactin levels are positively associated with mammographic density in postmenopausal women. Breast Cancer Res Treat. 2007; 105(3):337-346. [PubMed: 17260098]

6. Bremnes Y, et al. Endogenous sex hormones, prolactin and mammographic density in postmenopausal Norwegian women. Int J Cancer. 2007; 121(11):2506-2511. [PubMed: 17657735]

7. Maskarinec G, et al. IGF-I and mammographic density in four geographic locations: a pooled analysis. Int J Cancer. 2007; 121(8):1786-1792. [PubMed: 17520679]

8. Tamimi RM, et al. Endogenous sex hormone levels and mammographic density among postmenopausal women. Cancer Epidemiol Biomarkers Prev. 2005; 14(11 Pt 1):2641-2647. [PubMed: 16284390]

9. Walker K, et al. Premenopausal mammographic density in relation to cyclic variations in endogenous sex hormone levels, prolactin, and insulin-like growth factors. Cancer Res. 2009; 69(16):6490-6499. [PubMed: 19679547]

10. McCormack VA, et al. Sex steroids, growth factors and mammographic density: a cross-sectional study of UK postmenopausal Caucasian and Afro-Caribbean women. Breast Cancer Res. 2009; 11(3):R38. [PubMed: 19545414]

11. Pettersson A, et al. Nondense mammographic area and risk of breast cancer. Breast Cancer Res. 13(5):R100. [PubMed: 22017857]

12. Lokate M, et al. Mammographic density and breast cancer risk: the role of the fat surrounding the fibroglandular tissue. Breast Cancer Res. 1186; 13(5)

13. Hoffmann T, Penel C, Ronin C. Glycosylation of human prolactin regulates hormone bioactivity and metabolic clearance. J Endocrinol Invest. 1993; 16(10):807-816. [PubMed: 8144855]

14. Sinha YN. Structural variants of prolactin: occurrence and physiological significance. Endocr Rev. 1995; 16(3):354-369. [PubMed: 7671851]

15. Gout PW, Beer CT, Noble RL. Prolactin-stimulated growth of cell cultures established from malignant Nb rat lymphomas. Cancer Res. 1980; 40(7):2433-2436. [PubMed: 6992985]

16. Tworoger SS, et al. Bioactive prolactin levels and risk of breast cancer: a nested case-control study. Cancer Epidemiol Biomarkers Prev. 2014

17. Schernhammer ES, et al. Circulating levels of insulin-like growth factors, their binding proteins, and breast cancer risk. Cancer Epidemiol Biomarkers Prev. 2005; 14(3):699-704. [PubMed: 15767352]

18. Tworoger SS, Sluss P, Hankinson SE. Association between plasma prolactin concentrations and risk of breast cancer among predominately premenopausal women. Cancer Res. 2006; 66(4):24762482. [PubMed: 16489055]

19. Schernhammer ES, Hankinson SE. Urinary melatonin levels and postmenopausal breast cancer risk in the Nurses' Health Study cohort. Cancer Epidemiol Biomarkers Prev. 2009; 18(1):74-79. [PubMed: 19124483]

20. Hankinson SE, et al. Circulating concentrations of insulin-like growth factor-I and risk of breast cancer. Lancet. 1998; 351(9113):1393-1396. [PubMed: 9593409]

21. Schernhammer ES, et al. Insulin-like growth factor-I, its binding proteins (IGFBP-1 and IGFBP-3), and growth hormone and breast cancer risk in The Nurses Health Study II. Endocr Relat Cancer. 2006; 13(2):583-592. [PubMed: 16728584] 
22. Rosner B. Percentage points for a generalized ESD many-outlier procedure. Technometrics. 1983; 25:165-172.

23. Clevenger CV, et al. Expression of prolactin and prolactin receptor in human breast carcinoma. Evidence for an autocrine/paracrine loop. Am J Pathol. 1995; 146(3):695-705. [PubMed: 7534043]

24. Rosner B, et al. Determination of blood pressure percentiles in normal-weight children: some methodological issues. Am J Epidemiol. 2008; 167(6):653-666. [PubMed: 18230679]

25. Tamimi RM, et al. Endogenous Sex Hormone Levels and Mammographic Density among Postmenopausal Women. Cancer Epidemiol Biomarkers Prev. 2005; 14(11):2641-2647. [PubMed: 16284390]

26. Emerman JT, et al. Elevated growth hormone levels in sera from breast cancer patients. Horm Metab Res. 1985; 17(8):421-424. [PubMed: 4054832]

27. Maddox PR, Jones DL, Mansel RE. Prolactin and total lactogenic hormone measured by microbioassay and immunoassay in breast cancer. Br J Cancer. 1992; 65(3):456-460. [PubMed: 1558804]

28. Missmer SA, et al. Reproducibility of plasma steroid hormones, prolactin, and insulin-like growth factor levels among premenopausal women over a 2- to 3-year period. Cancer Epidemiol Biomarkers Prev. 2006; 15(5):972-978. [PubMed: 16702379]

29. Hankinson SE, et al. Reproducibility of plasma hormone levels in postmenopausal women over a 2-3-year period. Cancer Epidemiol Biomarkers Prev. 1995; 4(6):649-654. [PubMed: 8547832] 


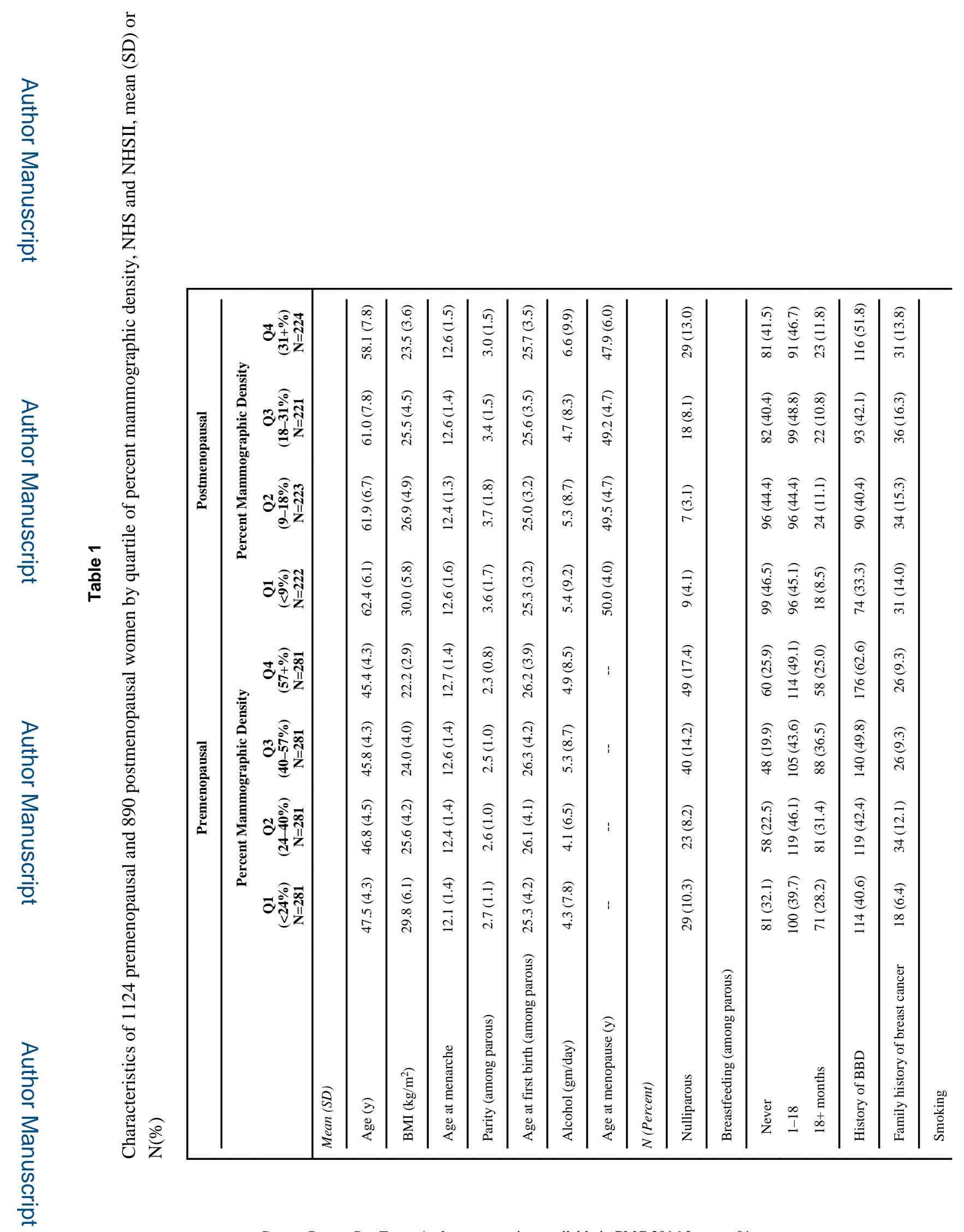

Breast Cancer Res Treat. Author manuscript; available in PMC 2016 January 01. 


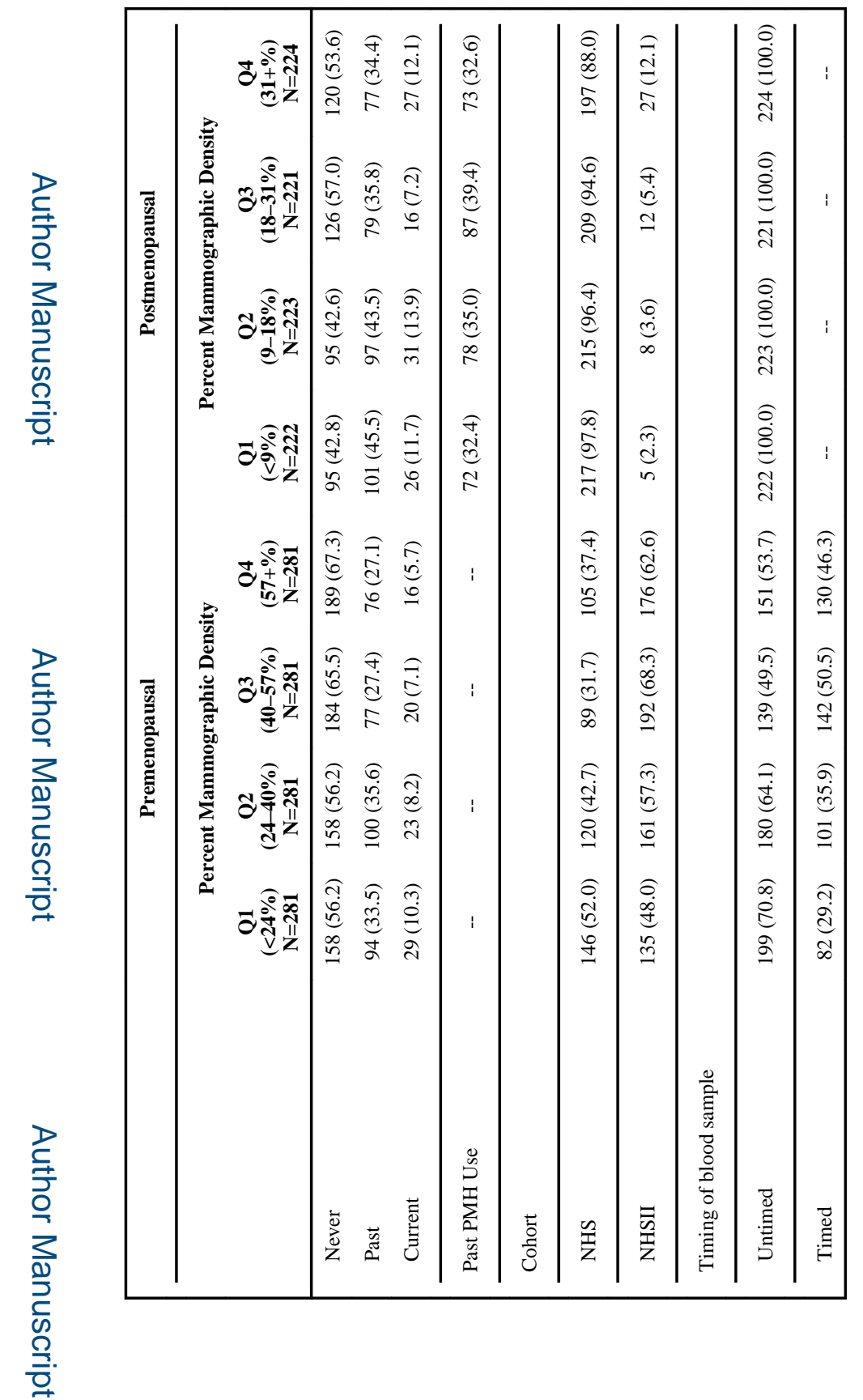

Breast Cancer Res Treat. Author manuscript; available in PMC 2016 January 01. 


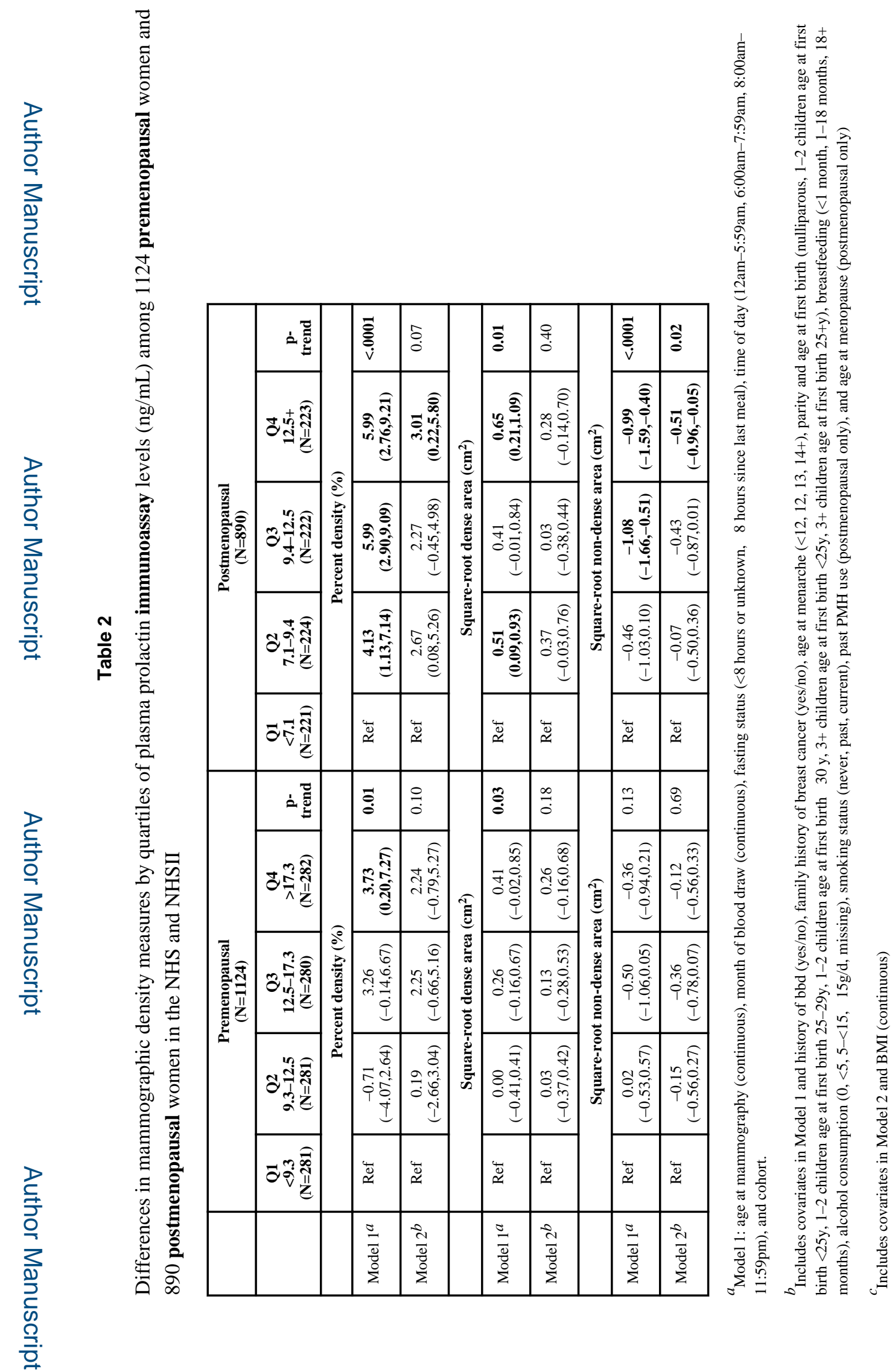

Breast Cancer Res Treat. Author manuscript; available in PMC 2016 January 01. 


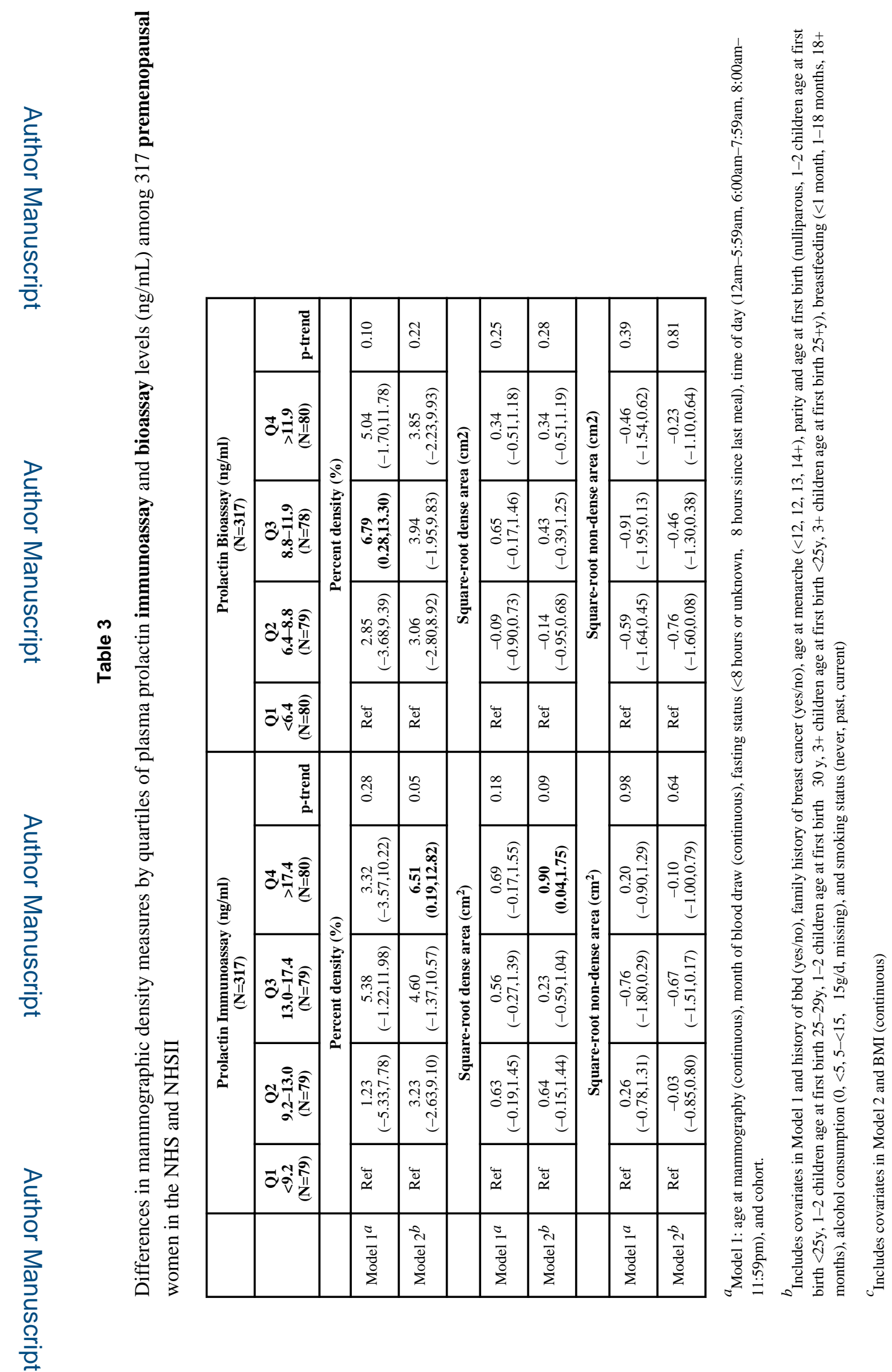

Breast Cancer Res Treat. Author manuscript; available in PMC 2016 January 01. 


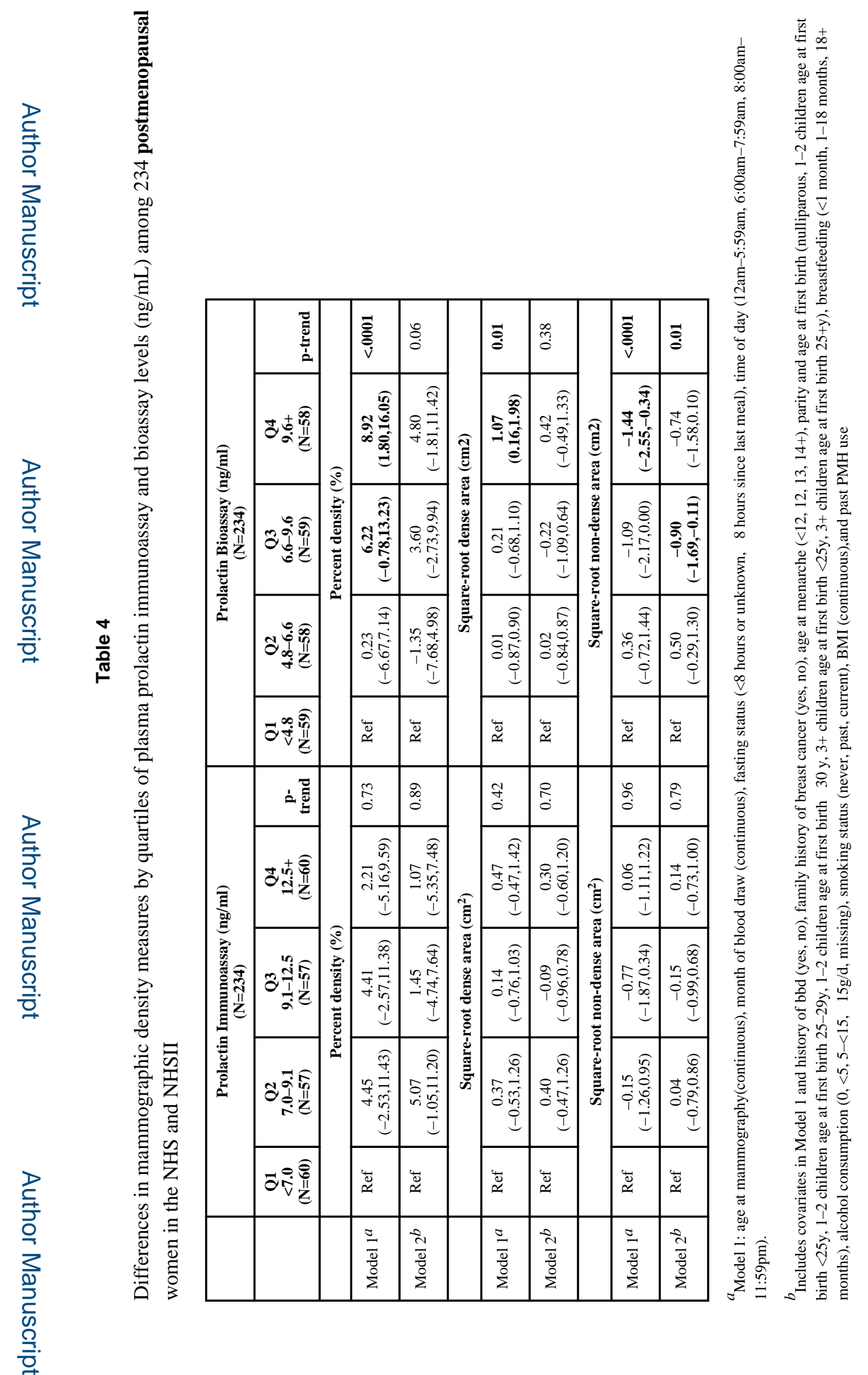

Breast Cancer Res Treat. Author manuscript; available in PMC 2016 January 01. 